\section{Diagnostic accuracy of nerve fibre layer, macular thickness and optic disc measurements made with the RTVue-100 optical coherence tomograph to detect glaucoma}

A Garas ${ }^{1}$, P Vargha² and G Holló

\begin{abstract}
Purpose To evaluate the diagnostic accuracy of retinal nerve fibre layer thickness (RNFLT), ganglion cell complex (GCC), and optic disc measurements made with the RTVue-100 Fourier-domain optical coherence tomography (OCT) to detect glaucoma in a Caucasian referral population.

Methods One randomly selected eye of 286 Caucasian patients (93 healthy, 36 ocular hypertensive, 46 preperimetric glaucoma, and 111 perimetric glaucoma eyes) was evaluated. Results Using the software-provided classification, for the total population sensitivity did not exceed $73.6 \%$ for the optic nerve head parameters, and $62.7 \%$ for the other parameters. Specificity was high (94.6-100\%) for most RNFLT and GCC parameters, but low (72.0-76.3\%) for the optic disc parameters.

Positive predictive value varied between 98.1 and $100 \%$ for the main RNFLT parameters, 92.6 and $100 \%$ for the 16 RNFLT sectors, 92.4 and $\mathbf{9 9 . 0 \%}$ for the GCC parameters, but did not exceed $86.3 \%$ for any of the optic disc parameters. Positive likelihood ratio (PLR) was higher than $\mathbf{1 0}$ for average, inferior and superior RNFLT (25.5 to infinite), 12 of the 16 RNFLT sectors (12.6 to infinite), and three of the four GCC parameters (40.0 to 48.6). No optic disc parameter had a PLR higher than 3.0. Conclusions RNFLT and GCC parameters of the RTVue-100 Fourier-domain OCT showed moderate sensitive but high specificity, positive predictive value and PLR for detection of glaucoma. The optic disc
\end{abstract}

parameters had lower diagnostic accuracy than the RNFLT and GCC parameters.

Eye (2011) 25, 57-65; doi:10.1038/eye.2010.139;

published online 8 October 2010

Keywords: diagnostic accuracy; glaucoma; optical coherence tomography; RTVue Fourier-domain OCT

\section{Introduction}

Compared with time-domain optical coherence tomography (OCT) with the third-generation Stratus OCT instrument (Carl Zeiss Meditec, Inc., Dublin, CA, USA) the recently developed Fourier-domain OCT technology provides several technical improvements that may increase its clinical usefulness for detection of glaucoma in clinical practice. ${ }^{1-6}$ In various studies, $^{7-12}$ the sensitivity and specificity of the Stratus OCT in detecting early glaucoma has been found to be only moderate to high. OCT instruments employing the Fourier-domain technology have significantly higher resolution and scan speed, improved image segmentation and better measurement reproducibility compared with the time-domain OCT. ${ }^{1-6}$ Though the technology is new, information on the diagnostic accuracy of the different Fourier-domain OCT instruments to detect glaucoma of different severity, is increasing., ${ }^{5,6,13-21}$

The RTVue-100 OCT (Optovue Inc., Fremont, CA, USA) is one of the new commercially available Fourier-domain OCT instruments. ${ }^{1-4,17-21}$ Its axial resolution is approximately $5 \mu \mathrm{m}$ and
${ }^{1}$ Department of

Ophthalmology, Semmelweis University, Budapest, Hungary

${ }^{2}$ Cardiovascular Centre, Semmelweis University, Budapest, Hungary

Correspondence: G Holló, Department of Ophthalmology, Semmelweis University, Budapest, Tömö u. 25-29 1083, Hungary Tel: + 3612100280 extn. 51627;

Fax: + 3612100309 E-mail: hg@szem1.sote.hu

Received: 27 May 2010 Accepted in revised form: 9 August 2010 Published online: 8 October 2010 
the scan speed is $26000 \mathrm{~A}$-scans per second. Thus the speed is 65 times higher than that of the Stratus OCT system, and the resolution is about twice as good as such time-domain OCT instruments. The RTVue optic nerve head map (ONH map) scan was developed for peripapillary retinal nerve fibre layer thickness (RNFLT) and two-dimension $\mathrm{ONH}$ measurements to detect glaucoma. As reduction of macular thickness, especially of the inner retinal layers, is an important OCT finding associated with glaucoma, ${ }^{7}$ the ganglion cell complex (GCC) scan of the RTVue system, which comprises tissue layers (the retinal nerve fibre layer, the retinal ganglion cell layer and the inner-plexiform layer) that are directly influenced by glaucomatous ganglion cell loss, may also have clinical importance. The instrument's software contains a normative database sufficient for statistical comparison for the different RNFLT, ONH and GCC parameters. $^{22}$

In this study, we investigated the diagnostic accuracy of the different RNFLT, GCC and ONH parameters of the RTVue-100 Fourier-domain OCT using the softwareprovided classifications for detection of glaucoma on 286 patients referred to our glaucoma centre over a period of 11 months.

\section{Materials and methods}

\section{Participants and patient groups}

The authors certify that all applicable institutional and governmental regulations concerning the ethical use of human volunteers were followed during this research. The research protocol was approved by the Institutional Review Board for Human Research of Semmelweis University, Budapest. Informed consent was obtained from all participants before enrolment. For inclusion, all participants had to have, in the study eye, sufficient central vision for optimal fixation, image quality sufficient for optimal evaluation, no macular pathology except for a small number of hard drusen, on stereoscopic evaluation. Of the 316 referred patients 30 were excluded from the study because of severe degenerative fundus changes in high ( $>14.0$ dioptres) myopia $(n=14)$, age-related macula degeneration, diabetic retinopathy and scar formation after vitreoretinal surgery $(n=7)$, non-glaucomatous optic neuropathies $(n=7)$ and cornea degeneration $(n=2)$. One randomly selected eye of each of the remaining 286 Caucasian individuals referred for detection or exclusion of glaucoma in the Glaucoma Centre of Semmelweis University in Budapest, who all underwent RNFLT, GCC, and ONH measurements made with the RTVue-100 Fourier-domain OCT between 1 January and 30 November 2009, was enroled in the study. All patients underwent the same diagnostic protocol, which comprised a detailed slit-lamp evaluation, stereoscopic $\mathrm{ONH}$ photography and evaluation by a glaucoma specialist (GH), stereoscopic evaluation of the macula, repeated Octopus normal or dynamic G2 threshold visual field testing, and daytime intraocular pressure phasing made with Goldman applanation tonometry within 2 months from the RTVue-100 OCT imaging. The final clinical classification based on the results of these tests was made by the head of the glaucoma team (GH). The RTVue-100 OCT examinations were made by a trained PhD student (GA), and were not used for the clinical classification of the patients.

The patient population comprised of 93 healthy subjects with no $\mathrm{ONH}$ damage, reliable and reproducible normal visual field tests with normal mean defect (MD), that is, MD less than $2 \mathrm{~dB}$, and intraocular pressure consistently below $21 \mathrm{~mm} \mathrm{Hg}$, based on daytime phasing (five measurements between 0745 and 1600 hours); 36 ocular hypertensive subjects with normal $\mathrm{ONH}$, visual field with MD less than $2 \mathrm{~dB}$ and untreated intraocular pressure consistently above $21 \mathrm{~mm} \mathrm{Hg}$; 46 preperimetric glaucoma patients characterized with definite glaucomatous neuroretinal rim loss (diffuse or localised neuroretinal rim thinning) and reliable and reproducible normal visual field with MD less than $2 \mathrm{~dB}$; and 111 perimetric glaucoma patients characterized with glaucomatous neuroretinal rim loss and reliable and reproducible visual field defect typical for glaucoma (inferior and/or superior paracentral or arcuate scotomas, nasal step, hemifield defect or generalised depression with MD higher than $2 \mathrm{~dB}$ ). Severity of glaucomatous visual field damage was classified according to the modified Bascom Palmer staging system..$^{23}$ The demographics of the participants are shown in Table 1.

\section{Fourier domain OCT}

OCT was performed through undilated pupil with the RTVue-100 Fourier-domain OCT instrument (Optovue Inc.) with software version 4.0. The working principle of the device has been described in detail elsewhere. ${ }^{1-4}$ In brief, the RTVue-OCT employs a near-infrared lightsource centred at $840 \mathrm{~nm}$, with a $50 \mathrm{~nm}$ bandwidth. The normative database for diagnostic classification consists of 861 healthy eyes of mixed ethnicity subjects, with ages ranging between 19 and 82 years. ${ }^{22}$ RNFLT values are found to correlate significantly with age of subject, ethnicity and with optic disc size, and adjustments for these effects (using multiple linear regression equations) are implemented in the software to improve classification results. For RNFLT, GCC and ONH measurements the standard glaucoma protocol was used. ${ }^{2}$ This includes 
Table 1 Demographic characteristics of the participants and eyes analysed in the study

\begin{tabular}{|c|c|}
\hline Race (n) & Caucasian (100\%) \\
\hline $\begin{array}{l}\text { Number of eyes involved in } \\
\text { statistical analysis }(n)\end{array}$ & $286(100 \%)$ \\
\hline Male/Female $(n / n)$ & $126 / 160$ \\
\hline $\begin{array}{l}\text { Best-corrected visual acuity } \\
(\text { mean } \pm \text { SD) }\end{array}$ & $0.9 \pm 0.2$ \\
\hline $\begin{array}{l}\text { Refractive error }(D) \\
(\text { mean } \pm S D \text {, range })\end{array}$ & $-0.7 \pm 2.9(-14.00+8.00)$ \\
\hline Prevalence of healthy eyes & $93 / 286(32.5 \%)$ \\
\hline Prevalence of OHT eyes & $36 / 286(12.6 \%)$ \\
\hline Prevalence of glaucoma eyes & $157 / 286(54.9 \%)$ \\
\hline Preperimetric & $46 / 157(29.3 \%)$ \\
\hline Perimetric & $111 / 157(70.7 \%)$ \\
\hline \multicolumn{2}{|l|}{ Type of glaucoma } \\
\hline Primary open-angle glaucoma & $103 / 157(65.6 \%)$ \\
\hline Juvenile open-angle glaucoma & $11 / 157(7.0 \%)$ \\
\hline Normal-pressure glaucoma & $12 / 157(7.6 \%)$ \\
\hline Chronic angle closure glaucoma & $11 / 157(7.0 \%)$ \\
\hline Pseudoexfoliative glaucoma & $7 / 157(4.5 \%)$ \\
\hline Pigment glaucoma & $9 / 157(5.7 \%)$ \\
\hline Other secondary glaucomas & $3 / 157(1.9 \%)$ \\
\hline Congenital glaucoma & $1 / 157(0.6 \%)$ \\
\hline \multicolumn{2}{|l|}{ Mean defect $(d B)($ mean $\pm S D)$} \\
\hline - Healthy eyes & $0.3 \pm 1.4$ \\
\hline - OHT eyes & $-0.1 \pm 1.2$ \\
\hline - Preperimetric glaucoma eyes & $0.1 \pm 1.8$ \\
\hline - Perimetric glaucoma eyes & $9.8 \pm 7.8$ \\
\hline \multicolumn{2}{|c|}{ Distribution of disease severity in the perimetric glaucoma group ${ }^{\text {a }}$} \\
\hline Stage 1 & $26 / 111(23.4 \%)$ \\
\hline Stage 2 & $34 / 111(30.6 \%)$ \\
\hline Stage 3 & $21 / 111(18.9 \%)$ \\
\hline Stage 4 & $24 / 111(21.6 \%)$ \\
\hline Stage 5 & $6 / 111(5.4 \%)$ \\
\hline \multicolumn{2}{|l|}{ Age (years) $($ mean $\pm S D)$} \\
\hline Healthy eyes & $54.9 \pm 15.9$ \\
\hline OHT eyes & $51.5 \pm 16.5$ \\
\hline Preperimetric glaucoma eyes & $57.6 \pm 11.8$ \\
\hline Perimetric glaucoma eyes & $62.2 \pm 14.7$ \\
\hline $\begin{array}{l}\text { Untreated maximal IOP of the OHT } \\
\text { eyes }(\mathrm{mm} \mathrm{Hg})(\text { mean } \pm \text { SD) }\end{array}$ & $29.1 \pm 8.7$ \\
\hline
\end{tabular}

Abbreviations: OHT, ocular hypertension; IOP, intraocular pressure.

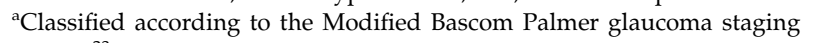
system. ${ }^{23}$

a 3D optic disc scan for the definition of the disc margin on the basis of the computer-assisted determination of retinal pigment epithelium endpoints, an $\mathrm{ONH}$ scan to measure the optic disc parameters and RNFLT within an area of diameter $4 \mathrm{~mm}$, centred on the pre-defined disc, and the standard GCC scan. Each ONH scan consists of 12 radial lines and six concentric rings, which are used to create an RNFLT map. The measuring circle (920 points) is derived from this map after the sample circle is adjusted to be centred on the optic disc. The measured RNFLT is automatically compared with the normative database for the total circle, the superior and inferior sectors, and each of the $1622.5^{\circ}$-sized sectors of the measuring circle. In the current investigation the following software-provided parameters were evaluated: (1) average RNFLT for the total $360^{\circ}$ around the ONH; (2) superior quadrant RNFLT; (3) inferior quadrant RNFLT; (4) all 16 separate RNFLT sectors (abbreviations: TU; temporal upper, ST; supero-temporal, SN; superonasal, NU; nasal upper, NL; nasal lower, IN; infero-nasal, IT; infero-temporal, and TL; temporal lower), (5) superior GCC (thickness of all macular layers between the internal limiting membrane and the inner plexiform layer, in the area above the horizontal meridian); and (6) inferior GCC (thickness of all macular layers between the internal limiting membrane and the inner plexiform layer, in the area below the horizontal meridian); (7) average GCC; (8) GCC focal loss volume (FLV; the total sum of statistically significant GCC volume loss divided by the GCC map area, in percent); (9) cup area; (10) cup/disc area ratio; and (11) rim area. For these software calculated parameters an instrument provided classification is indicated in a colour coded manner: sectors with 'within normal limits' classification (ie sectors for which the probability of there being no glaucomatous damage $\geqslant 5 \%$ ) are printed in green, sectors with 'borderline' classification $(P<5$ but $\geqslant 1 \%)$ in yellow and sectors with 'outside normal limits' classification $(P<1 \%)$ in red. In the current investigation both the retinal pigment epithelium endpoints and the $\mathrm{ONH}$ contour line were determined by the same trained examiner (AG) 4. Image quality was carefully checked after each image acquisition, and all images of insufficient quality or with any artefact were rejected and reacquired. To be included in the analysis, images had to have a signal strength index $>40$.

\section{Statistics}

The SPSS 15.0 program package was used for statistical analysis (SPSS Inc., Chicago, IL, USA). ANOVA with the Tukey post hoc tests was used to compare age and the measured parameter values between the patient groups. Sensitivity, specificity, positive predictive value, negative predictive value, positive likelihood ratio (PLR) and negative likelihood ratio of the software provided classification results were determined. $P$-values of $<0.05$ were considered as statistically significant.

\section{Results}

The age of the perimetric glaucoma group was higher than that of the healthy participants and ocular 
hypertensive group (Table 1 , ANOVA, $P<0.01$, Tukey post hoc test, $P<0.003$ for both comparisons). For the other comparisons no significant age difference was seen. All images met the pre-defined signal strength criterion and were analysed.

Comparison of the different RNFLT, GCC and ONH values between the patient groups is shown in Table 2 . Superior RNFLT and RNFLT sector ST 1 were significantly thinner for the ocular hypertensive eyes than the healthy eyes. For the other group comparisons most RNFLT, GCC and ONH parameters differed significantly between the groups, showing decreasing RNFLT, GCC thickness and rim area values, and increasing cup area and cup/disc area ratio with increasing disease severity categories.

Diagnostic performance of the software provided classification is shown in Table 3 , for each disease category and parameter, respectively. When borderline and outside normal limits classifications were grouped together (both considered abnormal), specificity was high (94.6-100\%) for most RNFLT and GCC parameters, and low $(72.0-76.3 \%)$ for the $\mathrm{ONH}$ parameters, in all analyses. For all parameters, sensitivity did not exceed $27.8 \%$ when discrimination of ocular hypertensive and healthy eyes was investigated. Sensitivity for detection of preperimetric glaucoma varied between 73.9 and $76.1 \%$ for the ONH parameters, but only between $6.5 \%$ (RNFLT sector NL1 and superior GCC) and $37.0 \%$ (superior RNFLT and RNFLT sector ST2) for the other parameters. For detection of perimetric glaucoma, GCC FLV showed the best sensitivity $(92.8 \%)$. Sensitivity of average RNFLT was $83.8 \%$. When discrimination of all preperimetric and perimetric glaucoma eyes from healthy eyes was evaluated, sensitivity varied between 66.2 and $69.4 \%$ for average RNFLT and the RNFLT quadrants, 17.2 and $71.3 \%$ for the RNFLT sectors, 52.2 and $72.0 \%$ for the GCC parameters, and 82.2 and $84.1 \%$ for the $\mathrm{ONH}$ parameters.

Considering the whole population (Table 4), the sensitivity values did not exceed $73.6 \%$ for the $\mathrm{ONH}$ parameters, and $62.7 \%$ for the other parameters. positive predictive value varied between 84.5 and $86.3 \%$ for the ONH parameters, and 92.4 and $100 \%$ for the RNFLT and GCC parameters. The highest negative predictive value value $(56.8 \%)$ was found for the $\mathrm{ONH}$ parameters. PLR was higher than 10 for average, inferior and superior RNFLT (25.5 to infinite), 12 of the 16 RNFLT sectors (12.6 to infinite), and three of the four GCC parameters (40.0-48.6). No ONH parameter had a PLR higher than 3.0. The negative likelihood ratio values varied between 0.4 and 0.9 . When borderline and within normal limits classifications were grouped together (all considered normal), specificity was similar to that in the other grouping, but sensitivity was considerably lower (detailed data are not shown).

\section{Discussion}

As detection of glaucomatous $\mathrm{ONH}$ damage is frequently suboptimal, ${ }^{24}$ evaluation of the diagnostic accuracy of the different imaging devices is of clinical importance. In the current study, we investigated the diagnostic capabilities of the RTVue-100 Fourier-domain OCT instrument on 286 Caucasian patients referred for glaucoma diagnostics to our centre during 11 months. The RTVue-100 OCT is one of the recently developed Fourier-domain OCT systems that all have several technical advantages compared with the time-domain OCT technology. ${ }^{1-6}$ Information on the clinical benefits provided by those technical improvements for detection of glaucoma and glaucoma progression, however, is limited. In most diagnostic accuracy studies, a healthy normal group and an age-matched glaucoma group with pre-defined disease severity are compared. ${ }^{6,11,14,15,17-21}$ In such investigations, for the best performing RNFLT and GCC parameters of the RTVue-100 OCT, the area under the receiver operating characteristic curve varied between 0.900 and $0.971 .{ }^{17-21}$ Other authors using other Fourier-domain OCT systems reported on similar values. ${ }^{6,15}$ These results suggest that under pre-defined circumstances the diagnostic accuracy of Fourier-domain OCT technology is somewhat higher than that of time-domain OCT technology. 6,11,15,20

Though using the above approach information on diagnostic capabilities of an instrument under pre-defined conditions can be specified, the clinical usefulness of the same instrument in unselected patient populations remains undetermined. In contrast, using data of all eyes successfully imaged during our nearly 1-year study period allowed us to evaluate diagnostic accuracy both for a general referral population and the different disease categories, separately. The significance of this approach is that disease severity may have an influence on the diagnostic capability of the Fourier-domain OCT instruments, ${ }^{16}$ thus it needs to be considered in the evaluation. But for routine clinical purposes, diagnostic accuracy determined for the total referral patient population is the most useful information.

To evaluate the diagnostic capability of the instrument we used the software-provided classification, which is based on comparison between the measured values and the integrated normative database. As the RTVue-100 OCT has an age and disc size adjusted separate database for Caucasians, ${ }^{22}$ which was used by us for our patients, the age-related RNFLT and GCC difference ${ }^{25,26}$ between our healthy control and ocular hypertensive subjects and the perimetric glaucoma patients was corrected for. We have previously shown that pupil dilation had no influence on the RNFLT and GCC measurements made 
Table 2 Comparison of the different retinal nerve fibre layer thickness (RNFLT), ganglion cell complex (GCC), and optic nerve head parameters between the patient groups.

\begin{tabular}{|c|c|c|c|c|c|c|c|c|c|c|c|c|c|c|}
\hline & \multicolumn{2}{|c|}{ Healthy (0) } & \multicolumn{2}{|c|}{ OHT (1) } & \multicolumn{2}{|c|}{$\begin{array}{l}\text { Preperimetric } \\
\text { glaucoma (2) }\end{array}$} & \multicolumn{2}{|c|}{$\begin{array}{c}\text { Perimetric } \\
\text { glaucoma (3) }\end{array}$} & \multicolumn{6}{|c|}{ P-values ${ }^{\mathrm{a}}$} \\
\hline & Mean & $S D$ & Mean & $S D$ & Mean & $S D$ & Mean & $S D$ & 0 vs 1 & 0 vs 2 & 0 vs 3 & 1 vs 2 & 1 vs 3 & 2 vs 3 \\
\hline \multicolumn{15}{|c|}{ Main RNFLT parameters ( $\mu \mathrm{m})$} \\
\hline Average & 106.5 & 9.1 & 102.0 & 10.7 & 94.9 & 12.3 & 74.9 & 12.0 & 0.173 & $<0.001$ & $<0.001$ & 0.021 & $<0.001$ & $<0.001$ \\
\hline Temporal & 77.9 & 10.4 & 76.8 & 10.2 & 69.9 & 9.7 & 55.8 & 13.4 & 0.964 & 0.001 & $<0.001$ & 0.039 & $<0.001$ & $<0.001$ \\
\hline Superior & 132.8 & 15.1 & 123.5 & 15.8 & 114.3 & 19.5 & 91.8 & 16.7 & 0.023 & $<0.001$ & $<0.001$ & 0.062 & $<0.001$ & $<0.001$ \\
\hline Nasal & 80.0 & 10.2 & 77.1 & 13.3 & 75.3 & 11.5 & 60.1 & 11.9 & 0.572 & 0.107 & $<0.001$ & 0.895 & $<0.001$ & $<0.001$ \\
\hline Inferior & 135.1 & 14.5 & 130.7 & 19.9 & 120.2 & 18.6 & 92.1 & 15.9 & 0.518 & $<0.001$ & $<0.001$ & 0.024 & $<0.001$ & $<0.001$ \\
\hline \multicolumn{15}{|l|}{ RNFLT sectors ( $\mu m)$} \\
\hline TU1 & 69.1 & 10.0 & 68.8 & 8.3 & 63.0 & 9.3 & 48.6 & 16.0 & 0.999 & 0.034 & $<0.001$ & 0.164 & $<0.001$ & $<0.001$ \\
\hline TU2 & 95.1 & 15.3 & 91.0 & 12.0 & 81.6 & 15.4 & 65.0 & 18.5 & 0.590 & $<0.001$ & $<0.001$ & 0.046 & $<0.001$ & $<0.001$ \\
\hline ST2 & 134.5 & 21.2 & 125.3 & 17.6 & 113.4 & 24.2 & 88.6 & 21.4 & 0.128 & $<0.001$ & $<0.001$ & 0.062 & $<0.001$ & $<0.001$ \\
\hline ST1 & 148.0 & 21.0 & 133.9 & 24.6 & 126.8 & 21.0 & 95.4 & 20.2 & 0.004 & $<0.001$ & $<0.001$ & 0.428 & $<0.001$ & $<0.001$ \\
\hline SN1 & 123.8 & 19.0 & 116.6 & 24.1 & 107.0 & 23.9 & 89.4 & 18.1 & 0.270 & $<0.001$ & $<0.001$ & 0.143 & $<0.001$ & $<0.001$ \\
\hline SN2 & 124.9 & 17.8 & 118.1 & 15.6 & 109.8 & 21.6 & 93.6 & 18.3 & 0.244 & $<0.001$ & $<0.001$ & 0.179 & $<0.001$ & $<0.001$ \\
\hline NU2 & 97.2 & 13.6 & 92.0 & 19.0 & 90.7 & 14.9 & 71.0 & 15.2 & 0.309 & 0.084 & $<0.001$ & 0.978 & $<0.001$ & $<0.001$ \\
\hline NU1 & 69.8 & 9.5 & 67.8 & 14.7 & 65.3 & 13.7 & 51.4 & 11.7 & 0.809 & 0.147 & $<0.001$ & 0.786 & $<0.001$ & $<0.001$ \\
\hline NL1 & 66.9 & 9.7 & 64.2 & 13.0 & 63.0 & 10.8 & 50.6 & 11.0 & 0.601 & 0.192 & $<0.001$ & 0.954 & $<0.001$ & $<0.001$ \\
\hline NL2 & 86.3 & 14.1 & 84.5 & 13.6 & 82.4 & 13.6 & 67.6 & 14.1 & 0.918 & 0.415 & $<0.001$ & 0.904 & $<0.001$ & $<0.001$ \\
\hline IN2 & 117.8 & 18.3 & 114.6 & 18.6 & 107.1 & 20.5 & 92.3 & 16.4 & 0.802 & 0.006 & $<0.001$ & 0.244 & $<0.001$ & $<0.001$ \\
\hline IN1 & 135.8 & 21.7 & 130.7 & 27.9 & 121.0 & 24.9 & 94.7 & 20.5 & 0.655 & 0.002 & $<0.001$ & 0.220 & $<0.001$ & $<0.001$ \\
\hline IT1 & 152.7 & 18.9 & 145.6 & 30.3 & 134.2 & 24.0 & 95.0 & 22.5 & 0.397 & $<0.001$ & $<0.001$ & 0.113 & $<0.001$ & $<0.001$ \\
\hline IT2 & 134.1 & 21.1 & 131.7 & 23.0 & 118.5 & 24.6 & 86.4 & 16.8 & 0.931 & $<0.001$ & $<0.001$ & 0.021 & $<0.001$ & $<0.001$ \\
\hline TL2 & 85.6 & 16.4 & 85.1 & 17.4 & 77.4 & 14.3 & 62.1 & 13.8 & 0.997 & 0.016 & $<0.001$ & 0.111 & $<0.001$ & $<0.001$ \\
\hline TL1 & 61.6 & 8.6 & 62.3 & 10.4 & 57.6 & 7.1 & 47.6 & 13.2 & 0.988 & 0.160 & $<0.001$ & 0.199 & $<0.001$ & $<0.001$ \\
\hline \multicolumn{15}{|l|}{ GCC parameters } \\
\hline Average $(\mu \mathrm{m})$ & 97.9 & 6.7 & 95.3 & 7.0 & 91.8 & 7.0 & 75.0 & 12.8 & 0.817 & 0.536 & $<0.001$ & 0.989 & $<0.001$ & $<0.001$ \\
\hline Superior $(\mu \mathrm{m})$ & 98.3 & 7.2 & 95.3 & 8.9 & 92.0 & 8.4 & 74.3 & 14.9 & 0.521 & 0.010 & $<0.001$ & 0.546 & $<0.001$ & $<0.001$ \\
\hline Inferior $(\mu \mathrm{m})$ & 97.5 & 6.7 & 95.2 & 6.4 & 91.5 & 6.8 & 75.6 & 13.7 & 0.665 & 0.006 & $<0.001$ & 0.348 & $<0.001$ & $<0.001$ \\
\hline FLV (\%) & 1.0 & 1.6 & 1.9 & 2.8 & 1.4 & 1.4 & 7.9 & 4.3 & 0.369 & 0.826 & $<0.001$ & 0.884 & $<0.001$ & $<0.001$ \\
\hline \multicolumn{15}{|c|}{ Optic nerve head parameters } \\
\hline Cup area $\left(\mathrm{mm}^{2}\right)$ & 0.842 & 0.540 & 0.801 & 0.530 & 1.486 & 0.493 & 1.603 & 0.556 & 0.980 & $<0.001$ & $<0.001$ & $<0.001$ & $<0.001$ & 0.600 \\
\hline Cup/disc area ratio & 0.416 & 0.233 & 0.417 & 0.214 & 0.696 & 0.121 & 0.819 & 0.177 & 1.000 & $<0.001$ & $<0.001$ & $<0.001$ & $<0.001$ & 0.002 \\
\hline Rim area $\left(\mathrm{mm}^{2}\right)$ & 1.113 & 0.468 & 0.962 & 0.304 & 0.627 & 0.265 & 0.334 & 0.314 & 0.150 & $<0.001$ & $<0.001$ & $<0.001$ & $<0.001$ & $<0.001$ \\
\hline
\end{tabular}

Abbreviations: FLV, focal loss volume; RNFLT sectors: TU, temporal upper; ST, supero-temporal; SN, supero-nasal; NU, nasal upper; NL, nasal lower; IN, infero-nasal; IT, infero-temporal; TL, temporal lower.

${ }^{a}$ Tukey post hoc test (ANOVA $<0.01$ for all parameters).

with the RTVue OCT. ${ }^{2}$ Thus we did not dilate pupil for the measurements. This fits in with busy routine clinical practice, and increases the clinical applicability of the results.

As shown in Table 2 in the ocular hypertensive group, for most parameters the mean values suggested some damage, but the difference from the healthy group was significant only for two parameters. In contrast, for all other groups several parameters showed significant damage compared with the healthy eyes, and the measured values showed more damage for the more severe disease categories, respectively.
For the evaluation of the software provided classification, borderline and outside normal limits classifications were grouped together as abnormal results. Prevalence of glaucoma exceeded $50 \%$ in the study population (ie, the number of eyes with and without glaucoma was comparable), thus it was meaningful to calculate the predictive values in addition to sensitivity, specificity and PLR. Specificity was consistently high (94.6-100\%); sensitivity was poor for detection of ocular hypertension and preperimetric glaucoma, and moderate to good (up to 92.8\%) for detection of perimetric glaucoma. For our total 


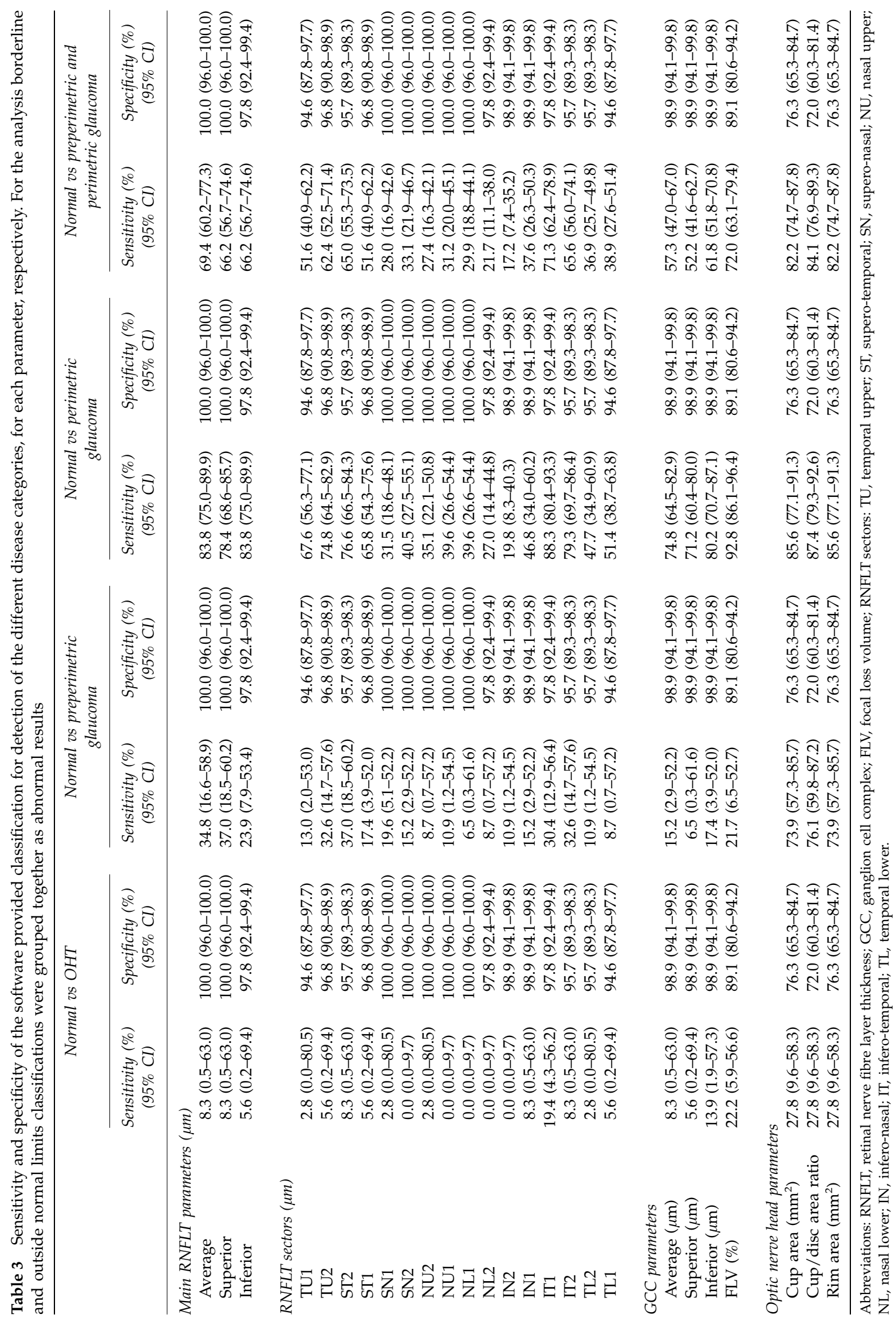


Table 4 Sensitivity, specificity, positive predictive value (PPV), negative predictive value (NPV), positive likelihood ratio (PLR) and negative likelihood ratio (NLR) of the software provided classification for detection of glaucoma in the total study population ( $n=286$ ), for each parameter, respectively. For the analysis borderline and outside normal limits classifications were grouped together as abnormal results

\begin{tabular}{|c|c|c|c|c|c|c|}
\hline & \multicolumn{6}{|c|}{ Normal vs OHT, preperimetric and perimetric glaucoma } \\
\hline & $\begin{array}{c}\text { Sensitivity (\%) } \\
(95 \% \text { CI) }\end{array}$ & $\begin{array}{c}\text { Specificity (\%) } \\
\quad(95 \% \text { CI) }\end{array}$ & $\begin{array}{l}P P V(\%) \\
(95 \% C I)\end{array}$ & $\begin{array}{l}N P V(\%) \\
(95 \% \quad C I)\end{array}$ & $\begin{array}{c}\text { PLR } \\
(95 \% \text { CI) }\end{array}$ & $\begin{array}{c}N L R \\
(95 \% \text { CI })\end{array}$ \\
\hline \multicolumn{7}{|c|}{ Main RNFLT parameters $(\mu m)$} \\
\hline Average & $58.0(48.8-66.8)$ & $100.0(96.0-100.0)$ & $100.0(96.7-100.0)$ & $53.4(43.4-63.2)$ & Infinite & $0.4(0.3-0.5)$ \\
\hline Superior & $55.4(46.0-64.5)$ & $100.0(96.0-100.0)$ & $100.0(96.5-100.0)$ & $52.0(41.9-61.8)$ & Infinite & $0.4(0.4-0.6)$ \\
\hline Inferior & $54.9(45.4-64.1)$ & $97.8(92.4-99.4)$ & $98.1(93.4-99.5)$ & $51.1(41.0-61.1)$ & $25.5(6.3-103.2)$ & $0.5(0.4-0.6)$ \\
\hline \multicolumn{7}{|l|}{ RNFLT sectors $(\mu m)$} \\
\hline TU1 & $42.5(32.4-53.3)$ & $94.6(87.8-97.7)$ & $94.3(87.0-97.6)$ & $44.2(34.3-54.6)$ & $7.9(3.2-19.7)$ & $0.6(0.5-0.7)$ \\
\hline TU2 & $51.8(42.1-61.4)$ & $96.8(90.8-98.9)$ & 97.1 (91.7-99.0) & $49.2(39.1-59.3)$ & $16.1(5.1-50.6)$ & $0.5(0.4-0.6)$ \\
\hline ST2 & $54.4(44.9-63.6)$ & 95.7 (89.3-98.3) & $96.3(90.8-98.6)$ & $50.3(40.1-60.4)$ & $12.6(4.7-34.2)$ & $0.5(0.4-0.6)$ \\
\hline ST1 & $43.0(32.9-53.7)$ & $96.8(90.8-98.9)$ & $96.5(90.1-98.8)$ & $45.0(35.1-55.3)$ & $13.3(4.2-42.5)$ & $0.6(0.5-0.7)$ \\
\hline SN1 & $23.3(13.4-37.5)$ & $100.0(96.0-100.0)$ & $100.0(92.1-100.0)$ & $38.6(29.3-48.7)$ & Infinite & $0.8(0.7-0.9)$ \\
\hline SN2 & $26.9(16.8-40.3)$ & $100.0(96.0-100.0)$ & $100.0(93.1-100.0)$ & $39.7(30.4-49.9)$ & Infinite & $0.7(0.6-0.9)$ \\
\hline NU2 & $22.8(12.9-37.1)$ & $100.0(96.0-100.0)$ & $100.0(92.0-100.0)$ & $38.4(29.2-48.6)$ & Infinite & $0.8(0.7-0.9)$ \\
\hline NU1 & $25.4(15.3-39.0)$ & $100.0(96.0-100.0)$ & $100.0(92.7-100.0)$ & $39.2(29.9-49.4)$ & Infinite & $0.7(0.6-0.9)$ \\
\hline NL1 & $24.4(14.3-38.2)$ & $100.0(96.0-100.0)$ & $100.0(92.4-100.0)$ & $38.9(29.6-49.1)$ & Infinite & $0.8(0.6-0.9)$ \\
\hline NL2 & $17.6(8.3-33.5)$ & $97.8(92.4-99.4)$ & $94.4(81.4-98.5)$ & $36.4(27.3-46.7)$ & $8.2(1.7-39.2)$ & $0.8(0.7-1.0)$ \\
\hline IN2 & $14.0(5.4-31.5)$ & 98.9 (94.1-99.8) & 96.4 (81.9-99.4) & $35.7(26.6-45.8)$ & $13.0(1.5-114.1)$ & $0.9(0.7-1.0)$ \\
\hline IN1 & $32.1(21.8-44.5)$ & 98.9 (94.1-99.8) & 98.4 (91.5-99.7) & $41.3(31.7-51.5)$ & $29.9(4.1-219.2)$ & $0.7(0.6-0.8)$ \\
\hline IT1 & $61.7(52.7-69.9)$ & $97.8(92.4-99.4)$ & 98.3 (94.1-99.5) & $55.2(44.9-65.0)$ & $28.7(7.1-115.5)$ & $0.4(0.3-0.5)$ \\
\hline IT2 & $54.9(45.4-64.1)$ & 95.7 (89.3-98.3) & 96.4 (90.9-98.6) & $50.6(40.4-60.7)$ & $12.8(4.7-34.5)$ & $0.5(0.4-0.6)$ \\
\hline TL2 & $30.6(20.3-43.2)$ & 95.7 (89.3-98.3) & $93.7(84.4-97.6)$ & $39.9(30.4-50.3)$ & $7.1(2.5-20.4)$ & $0.7(0.6-0.9)$ \\
\hline TL1 & $32.6(22.4-44.9)$ & $94.6(87.8-97.7)$ & $92.6(83.5-96.9)$ & $40.4(30.7-50.8)$ & $6.1(2.4-15.6)$ & $0.7(0.6-0.9)$ \\
\hline \multicolumn{7}{|l|}{ GCC parameters } \\
\hline Average $(\mu \mathrm{m})$ & $48.2(38.3-58.2)$ & 98.9 (94.1-99.8) & $98.9(94.2-99.8)$ & $47.6(37.7-57.8)$ & $44.3(6.2-318.3)$ & $0.5(0.4-0.6)$ \\
\hline Superior $(\mu \mathrm{m})$ & $43.5(33.4-54.2)$ & $98.9(94.1-99.8)$ & $98.8(93.6-99.8)$ & $45.5(35.7-55.7)$ & $40.0(5.6-288.6)$ & $0.6(0.5-0.7)$ \\
\hline Inferior $(\mu \mathrm{m})$ & $52.8(43.2-62.3)$ & 98.9 (94.1-99.8) & 99.0 (94.7-99.8) & $50.0(39.9-60.1)$ & $48.6(6.8-348.2)$ & $0.5(0.4-0.6)$ \\
\hline FLV (\%) & $62.7(53.8-70.8)$ & $89.1(80.6-94.2)$ & $92.4(86.2-95.9)$ & $53.2(42.5-63.7)$ & $5.8(3.1-10.9)$ & $0.4(0.3-0.5)$ \\
\hline \multicolumn{7}{|c|}{ Optic nerve head parameters } \\
\hline Cup area $\left(\mathrm{mm}^{2}\right)$ & $72.0(64.0-78.8)$ & $76.3(65.3-84.7)$ & $86.3(79.6-91.1)$ & $56.8(45.2-67.7)$ & $3.0(2.0-4.7)$ & $0.4(0.3-0.5)$ \\
\hline Cup/disc area ratio & $73.6(65.8-80.1)$ & $72.0(60.3-81.4)$ & $84.5(77.7-89.6)$ & $56.8(44.9-68.0)$ & $2.6(4.7-3.9)$ & $0.4(0.3-0.5)$ \\
\hline Rim area $\left(\mathrm{mm}^{2}\right)$ & $72.0(64.0-78.8)$ & $76.3(65.3-84.7)$ & $86.3(79.6-91.1)$ & $56.8(45.2-67.7)$ & $3.0(2.0-4.7)$ & $0.4(0.3-0.5)$ \\
\hline
\end{tabular}

Abbreviations: RNFLT, retinal nerve fibre layer thickness; GCC, ganglion cell complex; FLV; focal loss volume; RNFLT sectors: TU, temporal upper; ST, supero-temporal; SN, supero-nasal; NU, nasal upper; NL, nasal lower; IN, infero-nasal; IT, infero-temporal; TL, temporal lower; PPV, positive predictive value; NPV, negative predictive value; PLR, positive likelihood ratio; NLR, negative likelihood ratio.

unselected study population, most RNFLT and GCC measurements had high specificity and positive predictive value (92.4-100\%), and clinically useful PLR ( $>10$ to infinite). No such favourable findings were obtained for the $\mathrm{ONH}$ parameters (cup area, cup/disc area ratio and rim area), which suggests that the Fourier-domain technology did not overcome the problems of $\mathrm{ONH}$ classification with the time-domain OCT technology. ${ }^{9,12,27}$

Our results mean that in routine clinical practice a borderline or outside normal limits classification given for the main RNFLT parameters, RNFLT sectors (except for the temporal sectors) or GCC parameters by the instrument's software, strongly suggests that the eye has lost retinal nerve fibres and macular ganglion cells. In contrast, because of the relatively low sensitivity and weak negative likelihood ratio, a within normal limits classification cannot exclude glaucoma.

Our study has limitations. As our patients were all Caucasians, our results cannot be applied to other ethnic groups, nor do our findings provide any information about the clinical value of the non-Caucasian normative database of the instrument.

In conclusion, in our Caucasian referral population comprising healthy, ocular hypertensive, preperimetric and perimetric glaucoma patients referred for detection or exclusion of glaucoma, the RTVue-100 Fourier-domain OCT and its Caucasian normative database were found 
to be highly specific to detect glaucoma. The sensitivity to detect preperimetric glaucoma, however, was moderate. The overall best-performing parameter was average RNFLT, but several other RNFLT and GCC parameters had similarly favourable diagnostic accuracy. Diagnostic performance for the different $\mathrm{ONH}$ parameters was not as high as that for the RNFLT and GCC parameters.

\section{Summary}

What was known before

- Fourier-domain optical coherence tomography is technically superior to time-domain optical coherence tomography. Diagnostic accuracy of time-domain optical coherence tomography to detect glaucoma is moderate.

\section{What this study adds}

- The RTVue Fourier-domain optical coherence tomograph had moderate sensitivity, but high specificity, positive predictive value and positive likelihood ratio for detection of glaucoma in a Caucasian referral population. The optic disc parameters had lower diagnostic accuracy than the retinal nerve fibre layer and ganglion cell complex parameters.

\section{Conflict of interest}

Gábor Holló is an unpaid consultant of Optovue, Inc. and Carl Zeiss Meditec, Inc. The other authors declare no conflict of interest.

\section{Acknowledgements}

This work was supported in part by Hungarian National Health Grant (ETT) 001/2009 (Dr Holló).

\section{References}

1 González-García AO, Vizzeri G, Bowd C, Medeiros FA, Zangwill LM, Weinreb RN. Reproducibility of RTVue retinal nerve fibre layer thickness and optic disc measurements and agreement with Stratus optical coherence tomography measurements. Am J Ophthalmol 2009; 147: 1067-1074.

2 Garas A, Vargha P, Holló G. Reproducibility of retinal nerve fibre layer and macular thickness measurement with the RTVue-100 optical coherence tomograph. Ophthalmology 2010; 117: 738-746.

3 Garas A, Tóth M, Vargha P, Holló G. Comparison of repeatability of retinal nerve fibre layer thickness measurement made using the RTVue Fourier-domain optical coherence tomograph and the GDx scanning laser polarimeter with variable or enhanced corneal compensation. J Glaucoma 2010; 19(6): 412-417.

4 Garas A, Vargha P, Holló G. Automatic, operator-adjusted, and manual disc definition for optic nerve head and retinal nerve fibre layer measurements with the RTVue- 100 optical coherence tomograph. J Glaucoma 2010. E-pub ahead of print 29 April 2010, doi:10.1097/IJG.0b013e3181d787fd.

5 Moreno-Montañés J, Olmo N, Alvarez A, García N, Zarranz-Ventura J. Cirrus high-definition optical coherence tomography compared with Stratus optical coherence tomography in glaucoma diagnosis. Invest Ophthalmol Vis Sci 2010; 51: 335-343.

6 Leung CK, Cheung CY, Weinreb RN, Qiu Q, Liu S, Li H et al. Retinal nerve fibre layer imaging with spectral-domain optical coherence tomography: a variability and diagnostic performance study. Ophthalmology 2009; 116: 1257-1263.

7 Parikh RS, Parikh SR, Thomas R. Diagnostic capability of macular parameters of Stratus OCT 3 in detection of early glaucoma. Br J Ophthalmol 2010; 94: 197-201.

8 Parikh RS, Parikh S, Sekhar GC, Kumar RS, Prabakaran S, Babu JG et al. Diagnostic capability of optical coherence tomography (Stratus OCT 3) in early glaucoma. Ophthalmology 2007; 114: 2238-2243.

9 Li G, Fansi AK, Boivin JF, Joseph L, Harasymowycz P. Screening for glaucoma in high-risk populations using optical coherence tomography. Ophthalmology 2010; 117: 453-461.

10 Schrems WA, Mardin CY, Horn FK, Juenemann AG, Laemmer R. Comparison of scanning laser polarimetry and optical coherence tomography in quantitative retinal nerve fibre assessment. J Glaucoma 2010; 19: 83-94.

11 Wollstein G, Ishikawa H, Wang J, Beaton SA, Schuman JS. Comparison of three optical coherence tomography scanning areas for detection of glaucomatous damage. Am J Ophthalmol 2005; 139: 39-43.

12 Hong S, Ahn H, Ha SJ, Yeom HY, Seong GJ, Hong YJ. Early glaucoma detection using the Humphrey Matrix Perimeter, GDx VCC, Stratus OCT, and retinal nerve fibre layer photography. Ophthalmology 2007; 114: 210-215.

13 Jeoung JW, Park KH. Comparison of Cirrus OCT and Stratus OCT on the ability to detect localized retinal nerve fibre layer defects in preperimetric glaucoma. Invest Ophthalmol Vis Sci 2010; 51: 938-945.

14 Chang RT, Knight OJ, Feuer WJ, Budenz DL. Sensitivity and specificity of time-domain vs spectral-domain optical coherence tomography in diagnosing early to moderate glaucoma. Ophthalmology 2009; 116: 2294-2299.

15 Park SB, Sung KR, Kang SY, Kim KR, Kook MS. Comparison of glaucoma diagnostic capabilities of Cirrus HD and Stratus optical coherence tomography. Arch Ophthalmol 2009; 127: 1603-1609.

16 Leite MT, Zangwill LM, Weinreb RN, Rao HL, Alencar LM, Sample PA et al. Effect of disease severity on the performance of Cirrus spectral-domain OCT for glaucoma diagnosis. Invest Ophthalmol Vis Sci 2010; 51: 4104-4109.

17 Mori S, Hangai M, Sakamoto A, Yoshimura N. Spectraldomain optical coherence tomography measurement of macular volume for diagnosing glaucoma. J Glaucoma 2010. E-pub ahead of print 15 February 2010, doi:10.1097/ IJG.0b013e3181ca7acf.

18 Seong M, Sung KR, Choi EH, Kang SY, Cho JW, Um TW et al. Macular and peripapillary retinal nerve fibre layer measurements by spectral domain optical coherence tomography in normal-tension glaucoma. Invest Ophthalmol Vis Sci 2010; 51: 1446-1452.

19 Tan O, Chopra V, Lu AT, Schuman JS, Ishikawa H, Wollstein $\mathrm{G}$ et al. Detection of macular ganglion cell 
loss in glaucoma by Fourier-domain optical coherence tomography. Ophthalmology 2009; 116: 2305-2314.

20 Sehi M, Grewal DS, Sheets CW, Greenfield DS. Diagnostic ability of Fourier-domain $v$ s time-domain optical coherence tomography for glaucoma detection. Am J Ophthalmol 2009; 148: 597-605.

21 Li S, Wang X, Wu G, Wang N. Evaluation of optic nerve head and retinal nerve fibre layer in early and advance glaucoma using frequency-domain optical coherence tomography. Graefes Arch Clin Exp Ophthalmol 2010; 248: 429-434.

22 Sinai MJ, Garway-Heath DF, Fingeret M, Varma R, Liebmann JM, Greenfield $S$ et al. The role of ethnicity on the retinal nerve fiber layer and optic disc area measured with Fourier domain optical coherence tomography. Invest Ophthalmol Vis Sci 50: E-abstract 4785.

23 Mills RP, Budenz DL, Lee PP, Noecker RJ, Walt JG, Siegartel LR et al. Categorizing the stage of glaucoma from pre-diagnosis to end-stage disease. Am J Ophthalmol 2006; 141: 24-30.
24 Reus NJ, Lemij HG, Garway-Heath DF, Airaksinen PJ, Anton A, Bron AM et al. Clinical assessment of stereoscopic optic disc photographs for glaucoma: the European optic disc assessment trial. Ophthalmology 2010; 117: 717-723.

25 Budenz DL, Anderson DR, Varma R, Schuman J, Cantor L, Savell J et al. Determinants of normal retinal nerve fibre layer thickness measured by Stratus OCT. Ophthalmology 2007; 114: 1046-1052.

26 Sung KR, Wollstein G, Bilonick RA, Townsend KA, Ishikawa H, Kagemann L et al. Effects of age on optical coherence tomography measurements of healthy retinal nerve fibre layer, macula, and optic nerve head. Ophthalmology 2009; 116: 1119-1124.

27 Rao HL, Zangwill LM, Weinreb RN, Sample PA, Alencar LM, Medeiros FA. Comparison of different spectral domain optical coherence tomography scanning areas for glaucoma diagnosis. Ophthalmology 2010; 117(9): 1692-1699. 